\title{
THE USE OF THREE-DIMENSIONAL VISUALIZATION ON THE BASIS OF THE MOTIONPARALLAX3D TECHNOLOGY IN THE DEVELOPMENT OF COMPLEX TECHNICAL DEVICES AND TRAINING OF ENGINEERS
}

\author{
Konstantin Kharin, Sergey Kuvshinov \& Valentin Pryanichnikov
}
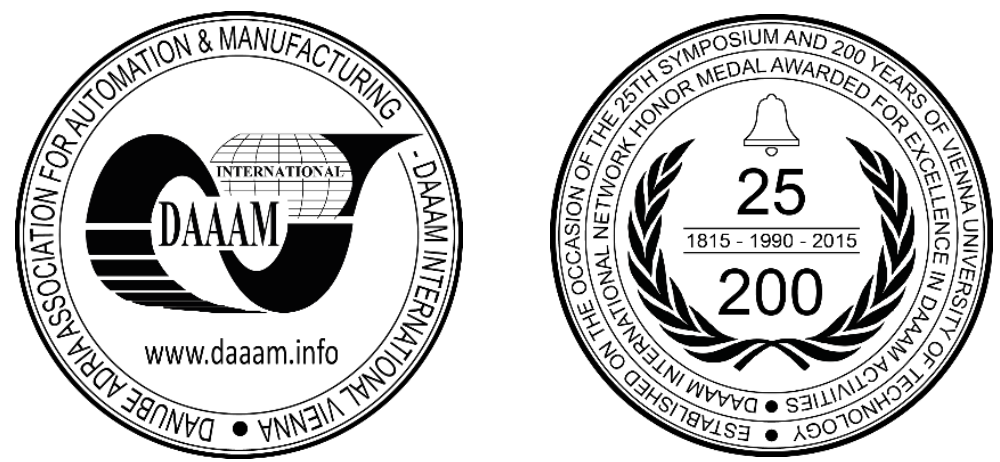

This Publication has to be referred as: Kharin, K[onstantin]; Kuvshinov, S[ergey] \& Pryanichnikov, V[alentin] (2016). The Use of Three-Dimensional Visualization on the Basis of the Motionparallax3D Technology in the Development of Complex Technical Devices and Training of Engineers, Proceedings of the 27th DAAAM International Symposium, pp.0338-0343, B. Katalinic (Ed.), Published by DAAAM International, ISBN 978-3-902734-08-2, ISSN 1726-9679, Vienna, Austria

DOI: $10.2507 / 27$ th.daaam.proceedings.049

\begin{abstract}
We consider some problems in the digitalization of modern engineering education and the need for improved clarity regarding the development of complex technical devices. A stereo display based on the MotionParallax3D technology is proposed for three-dimensional visualization of objects. The actual use of this technology in engineering education processes allows us to conclude that the students can rather quickly develop "three-dimensional" thinking, which increases the creativity of the entire educational process.
\end{abstract}

Keywords: engineering education; 3D modeling; three-dimensional visualization; MotionParallax3D technology.

\section{Introduction}

Engineering education is the basis for the development of modern society in the era of transition to a knowledge based society [1]. We realized that there is almost no means to develop three-dimensional thinking and growth the culture of work with three-dimensional models for future engineers. Three-dimensional visual technologies have become a foundation for building the structure of a new digital educational environment and arranging a new type of design, teaching and research, and, most importantly, production space [2]. The implementation of 3D technologies and digital personal production processes in educational institutions provides a unique opportunity for teachers to find new ways of handling with educational and scientific resources using the technology of case studies, particularly in a specially installed place (digital lab).

Due to the digital organization, these laboratories can efficiently use network technologies to create a distributed environment [3] for research and educational purposes [4]. It should also be noted that network technologies can be used to create educational and situational centers for these distributed laboratories with the help of modern three-dimensional visualization tools, such as 3D virtual studios [5]. However, it is modeling and prototyping that form the basis of the majority of advanced technologies and processes for the development of complex technical devices. The article considers the problem of determining the optimal composition of technical means to ensure the development of complete mathematical models of designed objects and the implementation of the respective learning network technologies. 
An important, if not the most important, methodological principle of modern design is thus the fact that the "designer" operates with simulating models and model systems, rather than the real processes and phenomena. In addition, the essence of real processes is judged from models. Probably, it can be stated that human reasoning is generally based on model images. Although universal models can hardly be found, the principle of modeling is obviously universal in any activity. With some reservations, a real end product of engineering can also be called a model.

In operating with models, the developers almost always need to visualize the model and analyze it as a virtual structure. In this context, the widespread use of digital visualization tools brings the development process to a new level. We demonstrate this on the example of the MotionParrallax3D technology.

One should especially note the fundamentally changed (in recent decades) approach to engineering and designing activities in general due to the widespread use of computer technologies, the focusing on digital production processes, and the significant speedup of design from the initial conception and sketching to the creation of first prototypes and their comprehensive analysis. An expert engineer should possess the following main skills:

- creative and critical thinking;

- analytical culture with knowledge from different fields;

- cooperation in international, intercultural, and interdisciplinary teams;

- independent learning in the age of rapid innovation and improvement of production processes and technologies as well as emergence of new materials;

- development of new ideas; advanced skills to handle with novel high-tech equipment and information systems under regular software updates, modification of regional and international standards for design, etc.

The modern workplace of design engineers, designers and developers involves a graphic workstation with professional software (CAD), which acts as both a "drawing stand" and a tool for the automation of computing associated with the stability and reliability of mechanisms and structures, and their coupling with other existing or projected units and elements of the finished product. The diversity of the equipment designed for working primarily with often threedimensional objects rather than with classical planar drawings puts forward new requirements for the visualization system in terms of efficient and most realistic visualization of these objects from different angles.

The technological chain of the project development from the model creation to the production of its prototype consists of three interconnected stages (Fig. 1). Here, the second stage (virtual prototyping) makes it possible to save time, materials, and equipment resources in the early stages of design.

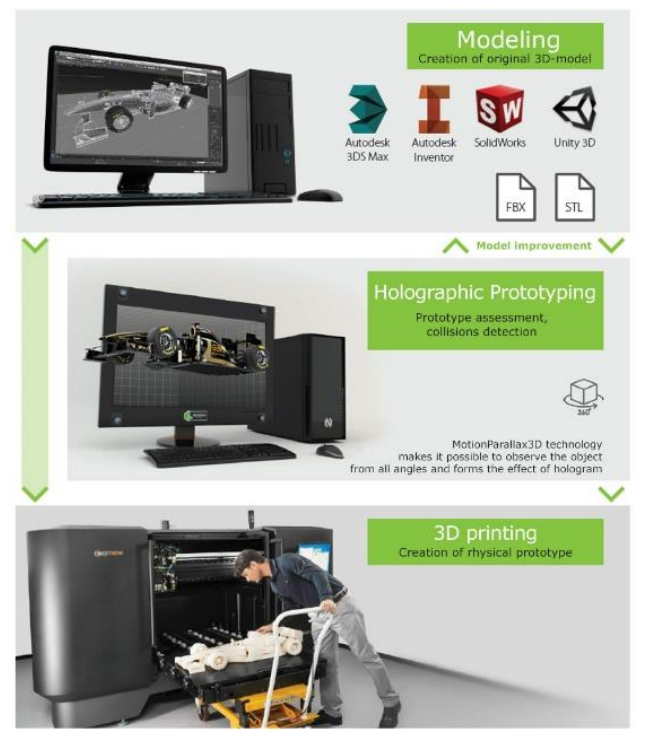

Fig. 1. Technological chain of modeling and prototyping. (1) Creation of original 3D-model using the known CAD and simulation packages. (2) Virtual prototyping. MotionParallax3D makes it possible to observe the object from all angles for a comprehensive assessment of the prototype, detection of collisions, design errors, reworking opportunities, etc. (3)

Creating a physical prototype by 3D-printing techniques

\section{The role and place of modeling and prototyping in the development of complex technical devices}

We must distinguish between two fundamentally different types of models — mathematical (reproducing and explaining the real mechanisms of object behavior) and imitation (describing only the basic interaction of an object as a "black box" with the outside world).

Despite the fact that the professional slang involves specific terms such as experimental, base, serial, etc., the above seems inappropriate in the design that is oriented to the end material product, where the product value is incommensurable to the values of the models that are auxiliary by definition. In the function-oriented design, the value (but not the cost) of all models is comparable, and this seems to be obvious. A possible example is the following fact of the history of the first- 
generation supersonic passenger aircraft created in the 1960s. American engineers actually triggered the development of Tupolev-144 and Concorde aircraft by the Russian and European aviation industries, respectively, but simultaneously they limit themselves to research on virtual models, which gave them a great experience and made it possible to save huge resources. If there arises a need in the near future for second-generation supersonic passenger aircraft, the United States will not have even potential competitors.

The study of models as an independent entity, unlike their practical use, has a relatively recent history; however, their typology is rather simple. The criteria may involve the language of description (presentation): from verbal to one that is implemented to actual product, the degree (depth) of engineering: from rough (unfinished) to detailed (finished), the functional purpose: from demonstration use to mass production, etc. Here, multiple criteria can be used for a single model.

A model is a universal tool for both representing and solving any problem. All design problems can be conditionally described (modeled) by a form defining the relations between entities under consideration, attributes, properties, and behavior (characteristics) in some external environment. The problem has one unknown parameter (entity, environment, or characteristics), which must be determined with the help of the other two components. Depending on the chosen unknown parameter, all problems can be divided into the following three main classes. The first class involves problems of analysis, when the entities are given and one analyzes the characteristics (unknown) that they have in a given external environment. The second class involves problems of synthesis, when the required characteristics are created and it is necessary to determine the entities that ensure these characteristics in a given environment. The third class involves problems of the external environment assessment, when the entities and their characteristics are given and it is necessary to obtain an environment (yet unknown), such that the given characteristics are manifested by the entities.

There is no formal method that would allow one to construct the most appropriate (correct) model for a given problem. During the solution, the problem of model construction and subsequent correction is imposed entirely on humans.

Simulation is an extremely complex, multi-faceted, and responsible process. It is particularly important that the unfinished model be correctly constructed at the stage of conceptual design; therefore, it makes sense to emphasize again that the construction of models in engineering design is to choose a way starting from some unfinished model and reaching a correct finished model.

Innovations in three-dimensional prototyping and improved digital production machines have made it possible to significantly reduce their cost and simplify the setup and overall activities. At present, the digital production laboratory is equipped with a set of computer-controlled universal tools with a possibility to operationally reconstruct the designed product. This outfit allows the laboratory to compete with mass production. In Europe and the United States, the laboratory has already proved to be capable of highly facilitating the development of single high-tech devices for specific purposes. The digital laboratory, where the product passes from its conception to working models, involves a system of audiovisual equipment: 3D video projectors, interactive visualization control tools (such as interactive panels, tables, and boards), three-dimensional sound systems; equipment for remote communication and high-quality multipoint video conferencing; digital production equipment: laser engraving machines, plasma cutters, knife cutters for cutting sheet materials, threeaxis CNC machines, computer-controlled subtractive milling and turning machines [6]; rapid prototyping tools: 3Dscanners for a rapid creation of very accurate three-dimensional models of existing components, objects, artifacts, their reengineering and decomposition and 3D-printers for a rapid creation of functional prototypes from the models using different technologies for various materials, such as plastic, photopolymers, metals, and paper.

These digital labs allow students to go from the conception to implementation of their ideas very quickly and, most importantly, obtain the result in a short time, rather than being fixed on presentations and computer models of products. The laboratory generates a new type of project thinking for young people. However, this does not occur immediately. The development of stereoscopic technologies allowing one to observe the real or virtual world in a more natural (threedimensional) form is only the first step. The technologies of 3D computer graphics that make it possible to simulate virtual objects (spaces), create three-dimensional models, and print them on 3D printers are the next step in the transition to the "three-dimensional" thinking.

The three-dimensional project perception has already been used in most activities, such as construction and design, simulation, and data analysis. Many elements of three-dimensional thinking have already been used in engineering, education, science, medicine, and culture. Relevant educational activities cover the fields of simulation, prototyping, and design. The students learn to think three-dimensionally (systemically) and create three-dimensional material objects. The process of three-dimensional thinking involves three-dimensional perception, three-dimensional simulation, 3D stereo training, interactive 3D stereo applications, development of intrinsic three-dimensional virtual objects, "materialization" of virtual reality, 3D printing of models, and, as a consequence, development of creative skills.

\section{The MotionParallax3D technology for technical objects modeling and prototyping}

An extension of the capabilities of currently widespread stereo monitors in terms of displaying three-dimensional models with a depth effect is the use of visualizing devices (with some conditionality, these can be called "holographic displays") in various stages; for example, we can mention the devices developed by the Russian company Nettle [7]. Alternatively, these devices can be called MotionParallax3D displays in view of the technology used [8].

We use the term "holographic" to suggest that the proposed visualization technologies make it possible to correctly and realistically (for humans) transmit the images of three-dimensional objects from different angles by analogy with holography, which has already become traditional for experts and where the images are recorded and displayed with the 
help of a highly stable laser light. In some sense, the virtual imaging on MotionParallax $3 D$ displays are close to multiplex holograms proposed by Lloyd Cross [9] but with a much more adequate demonstration of objects, which allows them to be regarded as full-fledged holograms in the direct sense of the word: as a complete optical information about the object.

Finally, the process of designing and prototyping can be characterized by the following key features and advantages:

- a high degree of detailing of models;

- demonstration of the details of any size and scaling;

- object decomposition, assembly/disassembly into elements;

- animation of the operation of units, possibility to observe the mechanism in the course of its operation;

- extended possibility for display: transfer of color and visual properties of materials (metal, plastic, etc.);

- presentation possibilities: the output model can be used in exhibition activities;

- distributed interaction between participants of the process: 3D-models can be sent electronically to remote areas of the world for evaluation;

- saving of time, materials, and equipment resources necessary for 3D-printing [10].

A multi-angle three-dimensional image makes an illusion of a solid object observable from all sides yet formed on a flat screen. In this case, the user is not separated from the real world: he can see both real and virtual objects with no difference in their behavior; the human brain fully believes in this illusion. In this sense, the proposed MotionParallax $3 D$ displays are superior to virtual reality helmets, which completely isolate the user from the real world, whereas the MotionParallax $3 D$ displays allow the user to be guided in the surrounding space to a greater or lesser degree. On the other hand, this imposes some restrictions on them: because the user can observe both real and virtual objects, their behavior should be identical. The projections of virtual objects are calculated so that the image observed by the user is fully identical to the image that would be observed if the virtual object was real and located at the appropriate point of the real space. The construction and mapping of correct projections of virtual objects requires that the virtual reality system be provided with the actual coordinates for observing the virtual world (the user's eye position).

Specialized 3D shutter-glasses with active trackers are used to allow the system to track the user's position in space with high accuracy. Based on eye position data, the system calculates the projection of the virtual world on the screen plane. Unlike stereo displays, which use only binocular vision, the MotionParallax $3 D$ displays use the three-dimensional perception mechanism of parallax motion, i.e., displacement of image parts relative to each other with an angular velocity that is proportional to the difference between them and the observer as their mutual location varies. This perception mechanism is activated by a permanent restructuring of the image according to the actual coordinates of the user's eyes. Due to this, the virtual objects are displaced relative to each other and relative to observable real objects according to the same laws and principles as the real-world objects. This allows the brain to build up a complete picture of the world containing both real and virtual objects with a visually indistinguishable behavior. However, due to the real-time projection realignment in this case, the objects are perceived as having some shape, volume and distance from the user's eyes, which, thus, are realistic from all angles.

Here, the image position and scale on the screen can be controlled both using a traditional computer "mouse" and in more intuitive ways: with a wireless touchpad, tablets and smartphones working under different mobile operating systems, gaming joysticks of different manufacturers or multi-axis 3D-manipulators.

The main focus in these devices is on the geometric correctness of the projection as the most significant indicator for the quality of the information mapping. The geometric correctness of projection is affected by the accuracy of user position tracking and the interval from the time when the user position is determined and the time when the image appears on the screen (the delay time). The tracking accuracy directly affects the correctness of the virtual object projection and can be determined from the general geometric error (depending on the architecture and geometry of tracking tools and the calibration quality) as well as the integral index of noise-induced error.

The tracking system uses unique Russian cameras with a scanning frequency of up to 900 frames per second in the normal mode. The four-camera system guarantees a one-millimeter accuracy and operational stability even in harsh illumination conditions. The working area of the tracking system with respect to vertical and horizontal (in the screen plane) viewing angles exceeds $170^{\circ}$. In this case, the optical tracking system can do without calibration during the operation, even after transportation of the equipment.

The interval from the time when the user position is determined and the time when the image appears on the screen (the delay time) is the main source of geometric incorrectness of the 3D-scene projection in MotionParallax $3 D$ systems. The delay is caused by the fact that all operations that determine the user's position as well as construct and display the projection require some time for their completion.

A characteristic feature of the technology of MotionParallax $3 D$ displays is the distributed processing of tracking data: each of the high-speed cameras has a built-in microcontroller with its own software based on Embedded Linux, which processes the data and transmits the marker position data that are ready for centralized processing. Also, to improve the quality of perception of virtual three-dimensional objects and significantly reduce the probability of above-mentioned symptoms for the user, the technology of user position prediction is used, which makes it possible to partially compensate for the delay; however, the prediction accuracy and horizon largely depend on many external factors. 


\section{The use of 3D visualization systems for educational purposes}

The visualization systems described above can be efficiently used in education through a ready-made content (primarily, in the disciplines of natural sciences) fully adapted to MotionParallax $3 D$ displays. Custom content can be created by a built-in development kit. The implementation of this technology in educational processes requires a special approach to the installation of classrooms. This full-featured computer class may include several workplaces with a full set of equipment and standard software as well as MotionParallax3D monitors for the interactive study from all angles, construction of models, and practical and creative work. The central workplace of the instructor makes it possible to control the models on the working systems of students. A 3D-projector and a projection screen or an interactive board allow individual angles of the chosen model to be broadcasted in the 2D or stereo (for viewing with glasses) modes to the entire audience (Fig. 2). The concept of this computer class is successfully integrated with the requirements for electronic simulators based on high-speed local networks for robotic laboratories [11].

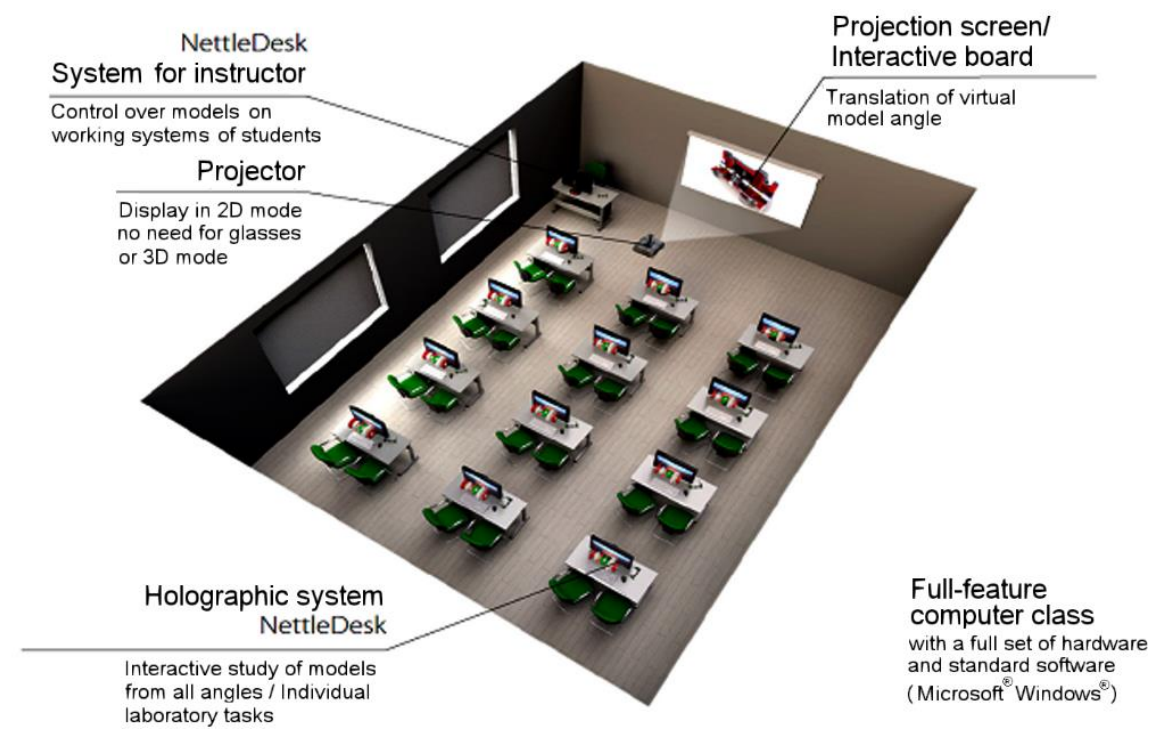

Fig. 2. Integrated solution for hardware of a holographic class

The ready-made educational content includes animated models of objects and phenomena from different fields, mostly causing problems in traditional teaching due to visualization complexities in conventional 2D graphics; for example, an internal combustion engine, the magnetic field of coil with current, different types of radiation, the heliocentric model of the world, the structure of gases, liquids and solids, and the principle of motor operation (in physics); different types of chemical bonds and the structure and form of electron clouds for electrons with different sets of quantum numbers (in chemistry); the structure of animal and plant cells, the process of photosynthesis, the structure of viruses and bacteria, and the structure of human eye (in biology).

Among the prospects of the proposed technologies, one can mention the potential increase in the number of users by increasing the frequency of stereo monitor frames for a correct separation of the video streams intended for each eye or by projecting the image on each eye individually with the help of special glasses. Also, it is important to simplify the integration of visualization systems with software for 3D-modeling or the creation of three-dimensional content in different fields that are not directly associated with digital technologies (including culture and the arts). It is of particular interest to develop devices for navigation and manipulation of virtual objects up to the user's full "immersion" into the resulting virtual world in educational and even entertainment purposes (Fig. 3).

\section{Conclusion}

The paper shows the way of selecting technical means for the implementation of efficient technologies for prototyping and modeling. For example, to intensify the process of training of future specialists, primarily in engineering, it is quite prospective and already widely used personal production technologies on the basis of laser cutting machines, 3D printers, plotters, CNC machines, etc. The objects have traditionally been visualized using 3D video projectors, stereoscopic monitors; however, a more qualitative display of a realistic three-dimensional picture of the object and a "presence effect" can be achieved by a successful application of the MotionParallax3D technology. The actual use of this technology in educational processes makes it possible to conclude that the students can rather quickly develop "three-dimensional" thinking, which sharply reduces the number of designing errors and inaccuracies. The new technologies of threedimensional mapping catalyze the transition of education from a reproductive level to a creative one. 


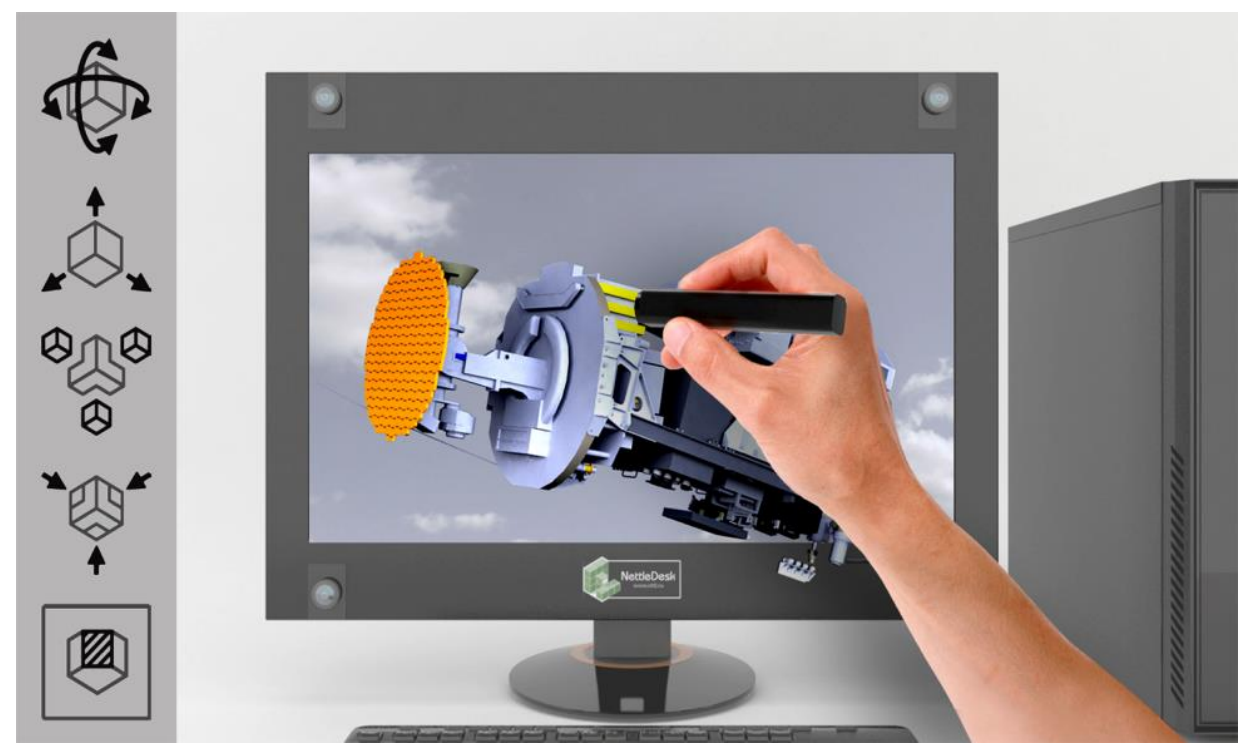

Fig. 3. Interactive manipulator for editing of virtual objects on a MotionParallax $3 D$ display

\section{References}

[1] Katalinic, B. (2010). Engineers for Knowledge Based Society, Annals of DAAAM for 2010 \& Proceedings of the 21st International DAAAM Symposium, 20-23rd October 2010, Zadar, Croatia, ISSN 1726-9679, ISBN 978-3901509-73-5, Katalinic, B. (Ed.), pp. 0001-0002, Published by DAAAM International Vienna, Vienna

[2] Kuvshinov, S.V. (2014). A technology of three-dimensional visualization for teaching in the humanities, in Recording and Viewing 3D Images in Motion Pictures and Other Fields: Proceedings of the VI International Conference, Moscow, April 17-18, 2014, Moscow: VGIK, 2014, pp. 239-245

[3] Pryanichnikov, V., Andreev, V., Bobrov, P., Biryukova, E., Frolov, A., Kharin, K., Kirsanov, K., Kostin, A., Kuvshinov, S., Marzanov, Y., and Prysev, E. (2012). Scientific-educational distributed laboratory - software and hardware means, in Annals of DAAAM for 2012 \& Proceedings of the 23th international DAAAM Symposium "Intelligent Manufacturing \& Automation" 24-27th October 2012 Zadar, Croatia, 2012, vol. 23, no. 1, pp. 11751178

[4] Andreev, V., Pryanichnikov, V., Poduraev, Y., and Kuvshinov, S. (2014). Education on the basis of virtual learning robotics laboratory and group-controlled robots, in 24th DAAAM Int. Symp. on Intelligent Manufacturing and Automation, 2013, Procedia Engineering, 2014, vol. 69, pp. 35-40

[5] Andreev V., Karbanov V., Kharin K., Kuvshinov S., Poduraev Y., and Pryanichnikov V. (2015). Training Situation Center Based on Three-Dimensional Virtual Studio for Distributed Mobile Robotics Laboratory, in Proceedings of the 26th International DAAAM Symposium "Intelligent Manufacturing \& Automation" 21-24th October 2015, Zadar, Croatia, EU, Ed. by B. Katalinic, 2015, Vol. 26, No. 1

[6] Kuvshinov, S.V. (2014). EduBrication: An innovative trend in European education, in Innovation Technologies in Motion Pictures and Education: Proceedings of Scientific and Practical Conference, Moscow, October 29-31, 2014, Moscow: VGIK, 2014, pp. 178-184

[7] http://www.rbc.ru/newspaper/2014/05/14/56beb80f9a7947299f72d0cc. (2014). Holographic millions, Accessed on: 2016-09-08

[8] https://en.wikipedia.org/wiki/MotionParallax3D. (2016). MotionParallax3D. Accessed on: 2016-08-30

[9] https://en.wikipedia.org/wiki/Holography. (2016). Holography. Accessed on: 2016-08-25

[10] Kuvshinov, S.V. and Uskov, G.N. (2013). The use of technologies of virtual reality and integrated stereoscopic 3Dsystems in educational processes, Mezhdunar. Nauchn. Zh., 2013, no. 4, pp. 57-64

[11] Andreev, V.P., Kirsanov, K.B., Levinsky, B.M., Pryanichnikov, V.E., and Travushkin, A.S. (2009). Development of a classroom of electronic simulation training systems on the basis of high-speed LAN, Information-Measuring and Control Systems, 2009, vol. 7, no. 6, pp. 82-92 